\title{
Les écrits didactiques pour femmes et le double discours du désir au Moyen Âge
}

Didactic works for women and the ambivalent discourse on desire in the Middle Ages

\section{Elizabeth Kinne}

\section{OpenEdition}

\section{Journals}

Édition électronique

URL : https://journals.openedition.org/clio/9624

DOI : $10.4000 /$ clio.9624

ISSN : 1777-5299

Éditeur

Belin

Édition imprimée

Date de publication : 1 mai 2010

Pagination : 135-152

ISSN : 1252-7017

Référence électronique

Elizabeth Kinne, "Les écrits didactiques pour femmes et le double discours du désir au Moyen Âge », Clio. Femmes, Genre, Histoire [En ligne], 31 | 2010, mis en ligne le 28 mai 2010, consulté le 15 février 2023. URL : http://journals.openedition.org/clio/9624 ; DOI : https://doi.org/10.4000/clio.9624 


\section{Regards complémentaires}

\section{Les écrits didactiques pour femmes et le double discours du désir au Moyen Âge}

Elizabeth KINNE

La littérature didactique pour femmes, qui connut en France son apogée aux quatorzième et quinzième siècles, ne paraît pas, au premier abord, un terrain fertile pour une étude de l'érôs. Les représentations de la vie conjugale que nous livrent Le Livre du Chevalier de la Tour Landry et Le Ménagier de Paris sont davantage des tableaux de privation que de volupté. Le lecteur averti y retrouvera en filigrane toute une tradition qui emprunte autant à la littérature courtoise, voire aux fabliaux et aux farces, qu'aux écrits ecclésiastiques. Il pourra y découvrir un discours foisonnant où sont déclinées des considérations sur la chasteté féminine, les désirs conjugaux, les pratiques quotidiennes et la hantise de l'adultère - féminin, s'entend.

Cette étude portera sur Le Livre du Chevalier de la Tour Landry pour ses filles et Le Ménagier de Paris, qui inventent ce qu'on pourrait appeler "une érotique de la didactique». La lecture de ces deux ouvrages semble d'abord confirmer le constat que la littérature didactique destinée aux femmes a pour ambition d'énoncer des formes de désir culturellement acceptables ${ }^{1}$. Double discours et double langage y

1 Voir l'introduction de Armstrong \& Tennenhouse 1987. 
expriment la hantise masculine d'un désir féminin illégitime en soi. Entre le non-dit et l'interdit se développe une esthétique érotique très particulière. Paradoxalement, là où le lecteur pourrait s'attendre à voir dévoilée une sexualité conjugale conforme à la morale, il ne trouve pratiquement que des représentations du désir adultère au fil de passages si scabreux qu'ils semblent plus aptes à donner des idées qu'à les corriger. Cependant, dans ces pages, les personnages féminins qui se livrent à ces actes en paient le prix fort; alors que le désir masculin se trouve du côté de la vie, le désir féminin relève d'une sémantique de la mort et de la souffrance.

Les discours prescriptifs sont néanmoins trompeurs. Ils dépeignent les fantasmes de deux narrateurs fictifs masculins, le chevalier et le ménager, qui semblent raviver les souvenirs de leurs conquêtes anciennes tout en instruisant des jeunes femmes afin qu'elles évitent de tomber dans de tels pièges ${ }^{2}$. Ils décrivent une utopie conjugale dans un récit qui est plus prescriptif que descriptif. Qui dit prescrire sous-entend obéir, mais les réactions des destinataires supposées à ces conseils ne nous sont pas connues. L'historien ne sait pas qui étaient les lectrices ou les éventuels lecteurs de ces ouvrages, ni comment ils mettaient en pratique les conseils qui y étaient donnés. Seul le recours à l'imagination permettrait de concevoir une éventuelle réponse féminine aux angoisses de ces narrateurs et de mesurer le degré de réalisation de ces prescriptions chez les couples bien réels de la même époque.

On pourrait plutôt qualifier cet érôs d'« anti-érôs » tant il prescrit d'interdictions. Les narrateurs y interdisent aux femmes toutes les pratiques sexuelles non procréatrices et extraconjugales car ce

2 Krueger a été parmi les premiers à observer cette contradiction interne: "The Chevalier $[\ldots]$ advocates on the one hand the containment, control, and surveillance of women in speech, dress, and social movement, and prohibits sexual autonomy. On the other hand, as a courtly knight who was once in love himself, he represents the tradition of the fin amant who idealizes women as objects of pleasure. Furthermore, his repeated accounts of women's sexual transgression reinscribe the very pleasures he eschews for his daughters, even as he heightens their horror with lurid descriptions of punishments. » (Krueger 1993 : 65). 
discours masculin voudrait réserver à l'homme le pouvoir d'instaurer, s'approprier, contrôler, et maitriser la sexualité du couple. Cependant, ces ouvrages témoignent d'une prise de conscience que le désir féminin échappe à celui qui voudrait désespérément en être le Pygmalion. Les auteurs ont alors recours à la menace, au surnaturel, car l'érôs pratiqué en dehors du lien matrimonial signifie la mort pour celle qui s'y livre, une mort qui peut être aussi bien réelle que spirituelle ou sociale. La femme qui transgresse est vouée à perdre son honneur, mettre en péril son rôle dans l'ordre social et spirituel.

\section{Variations autour de l'Érôs courtois}

Le Livre du Chevalier de la Tour Landry et Le Ménagier de Paris datent de la fin du quatorzième siècle, une époque de profondes mutations sociales et un moment où se développe une prédilection pour une littérature didactique qui se propose de représenter et redéfinir les rôles des acteurs socio-économiques. La littérature didactique, qu'il s'agisse des miroirs aux princes ou des ouvrages pour femmes, permettait de donner à comprendre comment «tenir son estat». Face à l'essor de la bourgeoisie urbaine et en pleine période de guerre avec une puissance extérieure, elle présentait un certain nombre de certitudes culturelles, sociales et économiques et illustrait leur mise en pratique, ce qui pouvait fournir aux lecteurs à la fois du réconfort et des outils pour asseoir leur place dans la société.

La situation des familles qui y sont décrites n'y est pas identique. Le Livre du Chevalier de la Tour Landry pour ses filles est supposé avoir été composé par un père de la petite noblesse pour l'éducation de sa progéniture féminine. L'auteur du Ménagier de Paris, qui dit être un bourgeois parisien, s'adresse à son épouse, une orpheline d'origine noble bien plus jeune que lui. Entre ces deux hommes âgés et les jeunes femmes qui en sont les destinataires, un rapport d'autorité s'établit dès le départ. Pour ces jeunes femmes, l'unique option est le rôle d'épouse, et tandis que le chevalier prépare ses filles à des mariages avec les nobles auxquels elles seront destinées, le bourgeois de Paris prépare son épouse à un mariage éventuel avec un deuxième époux au cas où il viendrait à mourir avant elle. 
En tant que précepteur masculin, comment parler de l'acte sexuel à des jeunes filles ? Comment parler de la chasteté, sans mentionner les relations sexuelles ? Certes, la doctrine chrétienne est claire sur ce point : chasteté absolue pour la jeune fille vierge, fidélité conjugale et sexualité maritale vouée uniquement à la procréation pour l'épouse, et, pour la veuve, sage abstinence dans le cadre d'un mariage spirituel ou remariage terrestre. Cependant, étant donné le grand intérêt qu'ils portent non seulement aux discours ecclésiastiques mais aussi aux genres littéraires profanes, nos auteurs se heurtent à des contradictions. En effet, les représentations de la sexualité au Moyen Âge relevaient de deux discours plus antagonistes que complémentaires. Tandis que l'amour courtois célébré dans les romans de chevalerie était un amour extraconjugal, les écrits ecclésiastiques se concentraient sur le rôle de la bonne épouse fidèle et soumise. Nos deux narrateurs se positionnent de façon contrastée vis-à-vis des valeurs courtoises, contraste qui est lié aux origines supposées des rédacteurs. Le Livre du Chevalier de la Tour Landry reprend peut-être plus facilement des éléments courtois car son auteur/narrateur est censé provenir de la petite noblesse alors que l'auteur/narrateur du Ménagier affirme son adhésion à la haute bourgeoisie urbaine. Leurs points de vue sur le topos courtois les différencient, mais ils arrivent néanmoins à un accord sur l'amour hors mariage: s'il est acceptable pour un homme, il est strictement interdit aux femmes. Cohérent, le Ménagier rejette les valeurs aristocratiques et repousse les amours illégitimes en dehors des possibilités d'une existence bourgeoise. Le narrateur du Ménagier trouve ainsi utile de prévenir son public des dangers que courent ceux qui fréquentent les fêtes galantes ou qui appartiennent à la cour des « grands seigneurs ». À sa jeune épouse, il explique :

Le . $v^{\mathrm{e}}$. article de la premiere distinction dit que vous devez estre tresamoureuse et tresprivé de vostre mary par-dessus toutes autres creatures vivans, moyennement amoureuse et privee de voz bons et parfaiz prochains parans charnelz et les charnelz de vostre mary, et tresestrangement privee de tous autres hommes; et du tout en tout estrange des oultrecuidez et oyseux jeunes hommes et qui sont de trop grant despence selon leur revenue, et qui sans terre ou grans lignages deviennent danceurs; et aussi des gens de court de trop grans seigneurs. 
En outre, de ceulx et celles qui sont renommez et renommees d'estre de vie jolye, amoureuse, ou dissolue. ${ }^{3}$

L'emprise du mari sur sa femme est autant une question de fidélité conjugale que de fidélité à sa classe sociale. «Gare à la cour!», dit en substance le ménager. En décrivant à sa jeune femme la hiérarchie des liens d'affection qu'elle doit entretenir avec les hommes autres que son mari et en excluant tout lien exogame, il inscrit l'amour conjugal dans un cadre social qui est hautement politique. Le débordement, la démesure et la discorde caractérisent les mœurs de ceux qu'il convient de ne pas fréquenter.

Si le narrateur du Ménagier de Paris peut facilement repousser les fréquentations indésirables hors du cadre du mariage bourgeois en les qualifiant tout simplement d'aristocratiques, le narrateur du Livre du Chevalier de la Tour Landry est face à un défi de taille. Comment définir un désir conjugal acceptable tout en puisant dans la tradition courtoise de la fin du Moyen Âge? Peut-on s'adresser à un public aristocratique en refusant certains aspects d'une tradition littéraire dont il est à la fois producteur et consommateur ? Faut-il docere ou delectare ou docere et delectare? En parlant de la sexualité, peut-on combiner l'utile et l'agréable alors que tout plaisir est formellement interdit?

Le narrateur du Livre du Chevalier se met en scène dans un jardin où il convoque ses souvenirs du temps où il était un jeune chevalier. En puisant dans la thématique de l'amour courtois, il explique qu'il souhaite instruire ses filles pour qu'elles évitent les pièges dans lesquels les jeunes filles peuvent risquer leur honneur. Cependant, un événement inhabituel survient dans la remémoration de ses amours de jeunesse. Alors qu'il chante les bienfaits de la dame qu'il aimait, la mort de celle-ci intervient brutalement.

Mes toux mes maulx me guerredonna pour ce que belle et bonne me donna, qui de honneur et de tout bien sçavoit et de bel maintien et de bonnes mœurs, et des bonnes estoit la meillour, se me sembloit, et la fleur. En elle tout me delitoye ; car en cellui temps je faisoye chançons, laiz et rondeaux, balades et virelayz, et chans nouveaux, le mieulx que je

3 Ménagier de Paris : 154. 
savoye. Mais la mort qui tous guerroye, la prist, dont mainte douleur en ay receu et mainte tristour. ${ }^{4}$

Dans son étude du Livre du Chevalier de la Tour Landry, Rebecca Barnhouse se demande si la dame en question est sa femme, Jeanne de Rougé5. S'agit-il d'une pure convention courtoise ? En fait, la mort de cette femme inconnue teintée de l'imagerie et du langage de la courtoisie fait écho aux morts réelles et symboliques des femmes évoquées dans les exemples qui suivent ce passage. L'élan amoureux du narrateur résulte de la mort inexpliquée de l'objet de ses affections, message important pour ses jeunes destinataires.

Si Le Livre du Chevalier de la Tour Landry débute avec un topos courtois, conforme à la fois à ses origines sociales et à ses prétentions littéraires, le spectre de la relation amoureuse extraconjugale qui hante la littérature didactique ne lui permet pas de rester longtemps fidèle aux exigences du genre ${ }^{6}$. Non seulement il prend en compte l'innocence de ses trois filles mais il porte un regard personnel distant vis-à-vis des comportements qu'il partageait avec ses compagnons quand il était plus jeune :

Et pour ce, quant je les vy vers moy venir, il me va lors souvenir du temps que jeune estoye et que avecques les compaignons chevauchoie en Poitou et en autres lieux. Et il me souvenoit des faiz et des diz que ilz me recordoient que ilz trouvoient avecques les dames et damoyselles que ilz prioient d'amours; car il n'estoit nulz jours que dame ou damoiselle peussent trouver que le plus ne voulsissent prier, et sy l'une n'y voulsist entendre, l'autre priassent sans attendre. Et se ilz eussent ou bonne ou male responce, de tout ce ne faisoyent-ilz compte; car paour ne honte n'en avoient, tant en estoient duiz et accoustumez, tant estoyent beaux langagiers et emparlez. Car maintes foiz vouloient partout desduit avoir, et ainsi ne faisoient que decevoir les bonnes dames et demoiselles, et compter partout les nouvelles, les unes vraies, les aultres mençonges, dont il en advint mainte honte et maint villain diffame sanz cause et sanz raison. ${ }^{7}$

4 Livre du Chevalier de la Tour Landry : 1-2.

5 Barnhouse arrive à la conclusion que c'est peu probable, car elle n'est morte qu'après 1383, bien après la date de rédaction que le narrateur nous donne comme étant 1371. Barnhouse $2006: 33$.

6 Pour une analyse détaillée du prologue voir De Gendt 2003 : 59-75.

7 Livre du Chevalier de la Tour Landry : 2-3. 
Si, comme l'affirme Georges Duby, l'amour courtois a été une manière de civiliser les hommes de la classe guerrière, le narrateur du Livre du Chevalier de la Tour Landry nous fait découvrir le revers de la médaille ${ }^{8}$. Les jeunes femmes qu'il dépeint ne sont pas des femmes avisées prêtes à céder peu à peu aux charmes de jeunes prétendants qui exprimeraient à la fois leur dévouement à la dame et, par personne interposée, à son mari. Celles qui se laissent envoûter par de beaux parleurs ou se prêtent au jeu de la séduction risquent leur réputation et encourent une mort éventuelle si elles laissent penser qu'elles en sont les complices.

Le lecteur averti retrouve dans cette anecdote les thèmes de l'errance chevaleresque et de la recherche du plaisir présents dans les romans courtois. Le narrateur laisse planer un léger doute sur la nature des jeux de séduction auxquels les jeunes chevaliers se livrent («ils eurent une bonne ou mauvaise réponse, ils n'en tenaient pas compte, car ils n'avaient ni peur, ni honte... »). Mais la violence et la menace du viol sont sous-entendues. À travers cette entrée en matière qui évoque la mort d'une femme aimée puis la violence à l'œuvre dans la conquête amoureuse, l'ouvrage s'annonce alors comme un outil propre à développer la capacité des jeunes filles à livrer bataille contre le désir des hommes voire contre leur propre désir?.

\section{Le confort conjugal, remède à l'adultère masculin}

$\mathrm{Si}$, pour la femme, le désir et la mort voisinent dangereusement, pour l'homme, l'érôs et la vie vont de pair.

Le Ménagier de Paris, ouvrage d'économie domestique par excellence, montre à quel point le confort bourgeois du foyer prime

8 Voir Duby $1990: 261-276$.

9 Le Chevalier aborde la question «d'aimer d'amour» dans un débat imaginaire avec son épouse vers la fin de l'ouvrage. Tandis que le narrateur soutient encore la part de l'amour courtois, la bonne épouse s'y oppose, évidemment. Le message que le Chevalier développe tout au long du livre se voit ainsi confirmé par une interlocutrice possédant une supériorité morale sur les trois destinataires supposées. Voir l'analyse de De Gendt sur ce passage et ses répercussions dans la littérature de la fin du Moyen Âge dans De Gendt 2003: 195-212. 
pour le mari. La femme qui ne le lui fournirait pas risquerait de le perdre et être ainsi « déconseillée » [désemparée] :

Sur quoy, belle seur, se vous avez autre mary apres moy, sachiez que vous devez moult penser de sa personne. [...] Et pour ce aymés la personne de vostre mary songneusement. Et vous pry que vous le tenez nectement de linge, car en vous en est. Et pour ce que aux hommes est la cure et soing des besongnes de dehors, et en doivent les mariz songner, aler, venir et racourir deça et dela par pluyes, par vens, par neges, par gresles, une foiz moullié, une foiz sec, une foiz suant, austresfoiz tramblant, mal peu, mal hebergié, mal chaussié, mal couchié - et tout ne luy fait de mal pour ce qu'il est resconforté de l'esperance qu'il a aux cures que la femme prendra de luy a son retour, aux aises, aux joyes et aux plaisirs qu'elle luy fera, ou fera faire devant elle : d'estre deschaux a bon feu, d'estre lavé les piez, avoir chausses et soullez fraiz, bien peu, bien abeuvré, bien servy, bien seignoury, bien couché en blans draps et cueuvrechiez blans, bien couvert de bonnes fourrures, et assouvy d'autres joyes et esbatemens, privetez, amours et secretz dont je me taiz. Et l'endemain robes-linges et vestemens nouveaulx. ${ }^{10}$

Le confort du foyer conjugal est aux antipodes des souffrances et des maux du monde extérieur. Les plaisirs corporels (avoir le corps au chaud, être propre, être vêtu bon linge) prennent place dans un espace dont le calme contraste avec l'agitation du monde extérieur.

Une autre anecdote, qui illustre l'art du conseil, permet de mesurer l'importance que revêt pour le narrateur du Ménagier le confort conjugal. Il s'agit de l'histoire de Mélibée et Prudence. Un jour, les ennemis de Mélibée entrent dans sa maison et blessent Prudence, sa femme, ainsi que leur fille. Mélibée enrage mais hésite entre soigner sa fille et appeler à la vengeance. Prudence l'encourage à assembler ses amis pour se faire conseiller et suivre la suggestion la plus avisée. Le narrateur du Ménagier fait l'éloge du comportement de Prudence à sa lectrice : «Et devez considerer les grans et cordialles pensees que luy en couvenoit avoir jour et nuyt a trouver si fors argumens et si vives raisons pour oster la rigueur de l'emprise a quoy son mary tendoit ${ }^{11}$ Le pouvoir qu'a l'épouse de calmer les esprits de son mari et le ramener dans le droit chemin est dépeint en des termes très valorisants. Cette histoire d'une femme qui donne conseil, même s'il

\footnotetext{
10 Ménagier de Paris : 294-296.
}

11 Ibid. : 400. 
ne s'agit que d'encourager son mari à suivre les conseils de ses amis masculins, invite la destinataire à agir de même ${ }^{12}$. Cependant, dans un autre exemple, la lectrice découvre une femme exemplaire, Jeanne la Quentine, que tout oppose à Prudence. Le contraste entre les deux femmes est passé sous silence et le narrateur tente de manière maladroite d'établir un parallèle entre la verve de Prudence, et l'humble soumission de Jeanne.

Jeanne la Quentine fait aussi preuve de patience et de diplomatie. Mais c'est avec des actes et non des paroles qu'elle conseille son mari. Lorsque Jeanne découvre que celui-ci la trompe avec une pauvre fileuse de laine, elle met au point une pieuse stratégie pour le regagner. L'auteur du Ménagier raconte :

Icelle dame Jehanne le souffry paciamment, et en la parfin enquist ou icelle povre fille demouroit, et tant enquist qu'elle le sceut, et vint en l'ostel et trouva la povre fille qu'il ne avoit aucune garnison quelzconques, ne de busches, ne de lart, ne de chandelle, ne de huyle, ne de charbon, ne de riens fors ung lit et une couverture, son thouret et ung pou d'autre mesnage. Si luy dist telz moctz: «M'amye, je suis tenue de garder mon mary de blasme, et pour ce que je scay qu'il prent plaisir en vous et vous ayme, et qu'il repaire ceans, je vous prye que de luy vous parliez en compagnie le moins que vous pourrez, pour eschever son blasme, le mien et de nos enfans, et que le celiez de vostre part; et je vous jure que vous et luy serez bien celez de moy. Car puis que ainsi est qu'il vous ayme, mon intencion est de vous aimer, secourir et aidier de ce que vous avrez a faire, et vous l'aparcevrez bien. Maiz je vous pry du cuer que son pechié ne soit revelé ne publyé. Et pour ce que je scay qu'il est de bonnes gens, qu'il a esté tendrement nourry, bien peu, bien pensé, bien chauffé, bien couché et bien couvert a mon pouoir, et que je voy que de luy bien aisier vous avez peu de quoy, j'ay plus cher que vous et moy le gardons en santé que je seule le gardasse malade. Si vous pry que vous l'amez et gardez et servez tellement que par vous il soit refraint et contregardé de villoter ailleurs en divers perilz. Et sans ce qu'il en sache riens, je vous envoyeray une grant paelle pour luy souvent laver les piez, garnison de busche pour le chauffer, ung bon lit, et duvet, draps et couverture selon son estat, cueuvrechiefs, orilliers, chausses et robeslinges nectes. Et quant je vous envoyeray des nectes, si me envoyez des

12 Pour une analyse des rapports entre l'histoire de Mélibée et Prudence et la littérature didactique, voir Collette 1995. 
sales, et que de tout ce qui sera entre vous et moy qu'il n'en sache rien, qu'il ne se hontoye $»^{13}$

L'inévitable s'ensuit. Le mari, curieux de la provenance des nouveaux biens de sa maitresse, l'accuse de malhonnêteté. La jeune femme, désemparée, lui raconte la vérité, et le mari honteux, retourne chez sa femme qui ne lui fait aucun reproche. Le lendemain, il va à la messe pour confesser ses péchés, et fait vœu de rester fidèle à son épouse.

Bien loin de la mise en scène courtoise, l'éloge du confort bourgeois et du pouvoir que peut exercer une épouse en assurant le confort de son mari y compris hors de son foyer est de mise dans cette anecdote. La destinataire peut ainsi observer la différence qui existe entre les valeurs de l'aristocratie et celles de la bourgeoise. L'épouse bourgeoise, parce qu'elle a le souci de son mari, est en droit d'intervenir dans l'ordre domestique de sa maitresse, par amour et par charité. Que Jeanne la Quentine, la bonne ménagère, soit prête à équiper la maison de la maitresse de son mari apparaît, de prime abord, comme une forme d'humilité mais c'est elle qui, finalement, prend le dessus. Le lavage des draps souillés par l'adultère lui permet d'exprimer sa dévotion conjugale et d'assainir ses relations conjugales. La propreté est une rédemption, l'humilité un pouvoir: voilà les leçons que la bonne ménagère doit retenir.

La bonne épouse se préoccupe davantage du corps de son mari que du sien propre. Lui fournir le confort et le protéger contre la maladie est encore de son devoir. Après avoir expliqué à la maitresse de son mari qu'elle préfère le partager en bonne santé que de l'avoir à elle toute seule malade, elle évoque les dangers qu'il pourrait encourir en dehors de la maison de sa maitresse : «Si vous pry que vous l'amez et gardez et servez tellement que par vous il soit refraint et

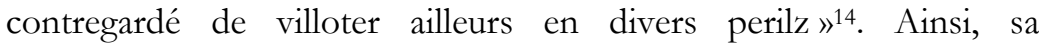
préoccupation pour le bien-être physique de son époux est un devoir que l'infidélité ne peut émousser. L'hygiène est un outil de reséduction mais est aussi représentative d'une pureté spirituelle. Ce sont ces attentions qui permettent la rédemption du mari adultère.

13 Ménagier de Paris : 402-404.

14 Ibid. : 402. 
Dans le Livre du Chevalier de la Tour Landry, les vertus de la dame de Languillier sont bien similaires à celles de Jeanne la Quentine. Toutes deux, face à l'adultère de leurs maris, manifestent de la patience et usent de leur savoir-faire domestique afin de les ramener dans le droit chemin. Cependant, la dame de Languillier se distingue par son statut social. Languillier est la femme noble d'un mari riche qui accueille des « folles femmes » sous son toit :

Celle bonne dame fut dame de Languillier, et avoit un seigneur qui tenoit bien mil et $\mathrm{v}^{\mathrm{e}}$ livres de rente, et tenoit moult noble estat. Et estoit le chevallier à merveille luxurieux, tant qu'il en avoit tousjours une ou deux à son hostel, et bien souvent il se levoit de delèz sa femme et aloit à ses folles femmes. Et, quant il venoit de folie, il trouvoit la chandoille alumée et l'eaue et le toaillon à laver ses mains. Et quant il estoit revenuz, elle ne ly disoit rien, fors qu'elle luy prioit qu'il lavast ses mains, et il disoit que il venoyt de ses chambres aisées : «Et pourtant, mon seigneur, que vous venès des chambres, avez-vous plus grant mestier de vous laver. » $\mathrm{Ne}$ autre ne lui reprouchoit, maiz que aucune foiz elle luy disoit privéement, à eulx tous deulx seulz : «Monseigneur, je sçay bien vostre fait de telle et telle. Maiz jà par ma foy, se Dieu plaist, puisque c'est vostre plaisir et que je n'y puis mettre autre remède, je n'en feray ne à vous ne à elles pire chière ne semblant. Car je seroys bien folle de tuer ma teste pour l'esbat de voz denrées, puisque autrement ne peut estre. Maiz, je vous prie, mon seigneur, que au mains vous ne m'en faciez point pire chière, et que je ne perde vostre amour ne vostre bon semblant; car du seurplus je me deporteray bien et en soufreray bien tout ce qu'il vous en plaira commander. » Et aucunes fois, par ces doulces parolles, le cuer lui en pitéoit et s'en gardoit une grant pièce. Et ainsi toute sa vie, par grant obéissance et par grant courtoisie le vainquoit ; car par autre voie jamaiz ne l'eust vaincu, et tant que au derrenier il s'en repentist et se chastia. Cy a bon exemple comment, par courtoisie et par obéissance l'on puet mieulx chastier et desvoyer son seigneur de celluy faict que par rudesse. Car il en est le plus de telz couraiges que, quant elles leur courent sus, ilz se appunaisissent et en font pis. ${ }^{15}$

Si la dame de Languillier exige de son mari qu'il respecte une règle d'hygiène pour établir un seuil entre la chaste chambre à coucher du couple et les espaces adultères impurs qui coexistent dans son foyer, elle enseigne par l'exemple à son mari un comportement modèle. Elle obtient la repentance de celui-ci et son retour dans le lit conjugal non parce qu'elle le châtie mais parce qu'elle se montre pure, et dans ses

15 Livre du Chevalier de la Tour Landry : 37-38. 
intentions et dans sa méthode éducative. En assimilant la satisfaction des désirs sexuels du mari au "bas" du corps (ce à quoi renvoient ses visites aux «chambres aisées»), le narrateur souligne l'impureté physique de son comportement, qui contraste avec la pureté physique et spirituelle de son épouse. Au contraire de son époux, celle-ci est tout à fait capable de se passer du « surplus » courtois, c'est-à-dire de l'assouvissement éventuel de ses propres désirs, afin de démontrer qu'elle a bien intégré la leçon qu'elle voudrait donner. Si, dans ces exemples, la sexualité masculine est active, la sexualité féminine est transmutée en domesticité active. La propreté du foyer agit comme une métaphore de la pureté morale qui assure la vie physique et spirituelle des maris repentis.

\section{La chasteté ou la mort}

Si les efforts que déploient les épouses pour prendre soin de leur mari lui pénètrent le corps et l'âme, le discours moralisateur qui est prononcé à cette occasion est censé pénétrer l'esprit féminin afin de lui permettre de maitriser son corps. Refuser ce discours revient à s'exposer à une pénétration mortelle par le pénis ou l'épée.

La violence contre les femmes forme la substance de bien des exemples didactiques. La menace du viol est particulièrement forte, ce qui fait présumer que, dans l'esprit des narrateurs, les hommes sont naturellement des agresseurs et que les jeunes filles victimes ne peuvent concevoir de céder leur chasteté que sous une menace de mort. Certaines, comme Lucrèce, se donnent même la mort de leur propre main ${ }^{16}$.

Cette relation entre l'acte sexuel et la mort est fort complexe. La mort guette la jeune fille ou l'épouse qui «cède» à la tentation de l'adultère (ou du moins, n'arrive pas à y résister de toutes ses forces). Par contre, les morts peuvent protéger les jeunes filles pieuses qui

16 «Adont Brut le conseillier et Colatin le mary d'icelle Lucresse, et tous ses amis, plourans et doulans, prindrent celle espee qui estoit sanglante, et sur le sanc jurerent par le sanc Lucresse que jamaiz ne fineroient jusques atant qu'ilz avroient Tarquin et son filz destruit ; et le poursuivront a feu et a sanc et a toute sa lignee boutee hors, si que jamaiz nul n'en vendra a dignité. » (Ménagier de Paris: 148). 
prient pour leur salut. Le Livre du Chevalier de la Tour Landry relate l'histoire des deux filles de l'empereur de Constantinople. L'une d'elle est très pieuse et prie constamment pour les morts, tandis que l'autre ne s'en soucie guère. Lorsque deux séducteurs entrent dans leurs chambres, la fille dévote n'est pas violée. Sa sœur, par contre, paie le prix fort de sa légèreté spirituelle :

Et quant celui qui devoit venir à la plus juenne cuida entrer entre les courtines, il lui sembla qu'il veist plus de mille hommes en suaires qui estoient environ la demoiselle. Si en eut si grant hideur et si grant paour qu'il en fut tous effrayez, dont la fièvre le prist et fut malades au lit. Maiz à l'autre chevalier ne avint pas ainsi, car il entra entre les courtines et ençainta la fille ainsnée de l'Empereur. Et quant l'Empereur sceut qu'elle fut grosse, il la fist noyer par nuit et le chevalier fist escorchier. Et ainsi par celui faulx delit morurent tous deux. ${ }^{17}$

La dévotion de la jeune fille définit l'impénétrabilité de son espace. Le chevalier qui la menaçait n'est pas arrivé à pénétrer ses courtines. L'autre en revanche, a accompli une triple pénétration : du lit de la fille, de son corps, et enfin, de sa lignée familiale. La seule solution pour l'empereur est de supprimer le maillon faible, la jeune fille qui ne suit pas à la lettre les conseils donnés, qui n'entre pas en lien spirituel avec cette même lignée familiale. De la même manière que la bonne ménagère peut étendre ou resserrer son domaine, la jeune fille doit assurer la garde de son propre corps. La femme est dotée de la faculté guerrière de contrôler les frontières de son espace procréateur, nouveau renversement des rôles traditionnels masculins et féminins. Tout comme Lucrèce, la jeune guerrière doit assurer une défense infaillible ou bien en souffrir les conséquences.

L'espace domestique où règne la femme reflète son corps, sa nature : la mauvaise femme ne peut assurer un espace sain, un foyer dédié à la propagation familiale. Dans une autre histoire qui concerne encore deux filles, en suivant le schéma moral binaire de la tradition des bonnes et mauvaises femmes, la Tour Landry esquisse le portrait d'une femme insuffisamment dévote et gourmande. Lorsque son mari la surprend dans le garde-manger avec leurs serviteurs en train de le

17 Livre du Chevalier de la Tour Landry : 7-8. 
vider, il la frappe, et elle devient borgne. Elle perd ainsi tout ce qu'elle devrait chérir :

Si luy messéoit trop à estre borgne, et la prist le seigneur en telle hayne qu'il se avilla et mist son cuer ailleurs, en telle manière que leur mesnage alla a perdicion du tout. [...] Sy avint que leur père, qui moult estoit proudomme, les ala veoir toutes deux; si trouva chiez l'une grans honneurs et grans richesses et y fut receu moult honnorablement, et chiez l'autre, qui avoit l'eueil trait, il y a trouva l'arroy et le gouvernement nice et malostru. ${ }^{18}$

Cette manière subtile de rappeler que la femme qui se comporte mal risque non seulement d'être défigurée et de perdre l'amour de son mari, mais aussi de perdre le respect de son père est un rappel à l'ordre patriarcal. Afin de bien tenir son ménage, une femme doit être vertueuse, car sans vertu elle ne peut garder les hommes socialement nécessaires au maintien de sa vie - ni son père, ni son mari.

Pour la femme, l'absence de maitrise du corps et l'acceptation d'une pénétration «indésirable » (soit alimentaire, soit sexuelle) signifient la négation d'un soi moral, social, et chrétien.

Évacuer le désir féminin, expression de la volonté individuelle, interdire la possibilité de son existence mettre la femme en position de n'être qu'un objet désiré qui reçoit la violence sexuelle ou physique, sert à asseoir une masculinité sexuelle et active. La lectrice reçoit ce dont on veut bien la remplir. Sujet malléable, elle est forcée d'accepter les failles de son instruction : objet sexuel, elle endosse à la fois les conséquences des actes qui sont commis sur elle et la culpabilité. Cette culpabilisation est à la fois une éducation politique et civique. Armstrong et Tennenhouse dans leur ouvrage sur la littérature didactique affirment que parler du désir est éminemment politique ${ }^{19}$.

Cependant, la femme n'est pas entièrement dépeinte comme étant passive dans le jeu de la séduction. Au contraire, elle doit bien orienter son aptitude à l'inaction dans une direction qui soit socialement et culturellement désirable : elle doit œuvrer à séduire l'homme que l'on veut bien lui donner, tout comme elle doit faire en

\footnotetext{
18 Livre du Chevalier de la Tour Landry : 13-14.

19 Armstrong \& Tennenhouse $1987: 2-3$.
} 
sorte de retransmettre un certain nombre de valeurs, d'en être l'illustration, d'assurer la propagation malgré elle. Cette ruse, qui fait apparaitre la volonté de l'autre comme la sienne propre, est la prémisse même de son instruction, mise en abyme du discours patriarcal, adhésion complète aux désirs masculins, et éducation politique. La jeune femme ne se voit pas alors interdire l'expression d'un désir, mais elle doit exprimer un désir de famille et non un désir sexuel. Ceci est un érôs politique, un stratagème qui en servant sa famille et son milieu social sert sa propre existence.

La récompense est, également, entièrement politique. Maintenir son statut social et perpétuer les valeurs de classe sont tout aussi importants que d'autres fonctions politiques liées au mariage. Le narrateur du Chevalier de la Tour Landry fait l'éloge de certains types de mariage, pourtant dénués d'intérêts économiques, qui sont axés sur la possibilité de perpétuer une lignée de valeurs, sinon, une lignée tout court.

Dont je vourroye que vous eussiez bien retenu l'exemple des filles du roy de Dannemarche. Si vous en compteray. Ilz sont quatre roys de çà la mer qui anciennement se marièrent par honnour, sans convoitise de terre, comme des filles de roys ou de haulx lieux, qui soient bien nées ou qui aient renommée de bonnes meurs, de bel maintien, et fermes, et de bonnes manières, et les convient veoir se elles ont ce que femmes doivent avoir et se elles sont tailliées de porter ligniée. ${ }^{20}$

La jeune femme qui adhère à ces préceptes maintient son statut ou bien l'améliore. Dans l'histoire des filles du roi de Danemark, qui sont mises à l'épreuve par un prétendant éventuel, celle qui réussit le mieux devient reine :

[...] car nulles gentilz femmes ne doivent avoir nul effroy en elles; elles doivent avoir gentilz cuers et de doulces responces et estre humbles, comme Dieu dist en l'Euvangille, que qui plus vault et scet plus se humilie, car qui plus se umilie plus s'essaulce, comme fist cest mainsnée fille du roy d'Arragon, qui, par sa courtoisie et son humilité, conquist a estre royne d'Espaigne et l'osta à sa suer l'ainsnée. ${ }^{21}$

Le vocabulaire guerrier, les verbes «conquérir» et «ôter», démontrent bien dans quel registre se situe la conquête amoureuse

20 Livre du Chevalier de la Tour Landry : 25.

21 Ibid. : 32. 
pour la femme. L'adhésion aux valeurs dominantes et leur intériorisation portent ses fruits.

Le narrateur du Ménagier de Paris tire une histoire du Moralitez sur le jeu des eschés, recueil très en vogue au quatorzième siècle. Le narrateur explique d'abord comment une bonne reine doit avant tout bien instruire ses filles et prendre garde à leur chasteté. Mais l'exemple de la duchesse Raymonde d'Italie, qui céda sa ville au roi Cantamus de Hongrie par luxure, démontre comment les filles peuvent se comporter encore mieux que leur mère. La duchesse, ayant trahi sa ville pour l'amour d'un des chevaliers de Cantamus, laisse y entrer son armée. Ses filles, horrifiées par la trahison de leur mère et prises de peur à l'idée d'être violées, ont caché des pigeons morts sous leurs seins : l'odeur repoussante met en fuite les agresseurs. Ainsi, en se souillant, elles sauvent leur virginité22. La duchesse, quant à elle, connait un sort particulièrement sordide :

Icellui chevalier print icelle duchesse et jeut avec elle une nuyt pour son serement sauver. L'endemain la fist a tous les Hongres commune. Le jour apres lui fist fichier ung peel des parmy la nature au long du corps jusques a la gorge, disant : «Tel mary doit avoir telle lecheresse qui par sa luxure a trahy sa cité et ses gens baillez es mains de leurs ennemis. » Et aussi ces parolles fist il escripre en plusieurs lieux parmy sa robe. Et toute morte la fist atachier et lier aux barrieres de deshors et devant la porte de sa cité, afin que chascun la veist, et la laissa. ${ }^{23}$

Le corps de la femme adultère devient un texte visible et lisible pour toute la cité dans cette anecdote qui nous montre, peut-être mieux que toutes les autres, le lien entre amour et mort, désir et politique, pour la femme qui n'agit pas comme il le faudrait. Tandis que nos narrateurs établissent une fraternité homo-sociale entre auteurs et lecteurs masculins, ils esquissent les contours d'une véritable bataille morale et spirituelle entre femmes, chacune ayant la responsabilité de faire preuve d'une bonne intégration de la leçon donnée au détriment de ses consœurs.

L'érôs didactique est avant tout un érôs de la négation, fondé sur le refus des pratiques sexuelles hors du cadre du mariage et de la

22 Ménagier de Paris : 140-142.

23 Ibid. : 142. 
reproduction. Il est, en même temps, une fantasmagorie qui ne correspond pas aux pratiques historiques, car elle est de nature prescriptive. Le texte didactique nous fournit la vision d'un narrateur qui se préoccupe de la manière dont la sexualité féminine devrait être orientée pour sa jouissance exclusive. Cette érotique didactique est une canalisation de l'érôs vers la civilisation, la projection de l'angoisse masculine vis-à-vis d'une paternité qui pourrait être incertaine et d'un honneur familial qui pourrait être flétri. Si le narrateur masculin écrit pour un public féminin, il écrit tout autant pour un public masculin capable de reconnaitre et d'apprécier son autorité morale et son habileté politique à diriger la plus petite cellule de la société, le foyer. L'assouvissement du désir sexuel n'existe pas dans le cadre du lien conjugal, mais le désir politique existe fortement.

La série d'anecdotes où sont relatées des relations sexuelles laisse entrevoir que la configuration de l'espace domestique s'effectue dans et à travers le corps féminin. La femme établit des frontières, des seuils et symbolise des territoires échangés entre hommes. Cet intérêt porté à l'espace dans les relations entre les hommes et les femmes établit une géographie politique érotisée. Mais elle est peut-être davantage orientée vers une géographie intérieure, celle de l'époux ou du père narrateur pris d'angoisse par rapport à la domination désirée de son foyer et son espace social. Dans la littérature didactique, la tentative de l'auteur de circonscrire les passions de l'autre féminin, d'imaginer sa mort inévitable, d'esquisser des frontières acceptables entre homme et femme, des seuils entre foyer et monde extérieur, devient une manière de garantir la pérennité d'un certain mode de vie et de certaines valeurs.

\section{Textes}

Le Livre du chevalier de la Tour Landry pour l'enseignement de ses filles, 1854, publié par M. Amable de Montaiglon, Paris, P. Jannet.

Le Mesnagier de Paris, 1994, texte édité par Georgina E. Brereton et Janet M. Ferrier, traduction et notes par Karin Ueltschi, Paris, Le livre de poche. 


\section{Bibliographie}

Armstrong Nancy \& Leonard Tennenhouse (eds), 1987, The Ideology of Conduct, New York, Methuen.

BARnhouse Rebecca, 2006, The Book of the Knight of the Tower: Manners for Young Medieval Women, New York, Palgrave Macmillan.

ColletTe Carolyn P., 1995, «Heeding the Counsel of Prudence: A Context for the Melibee », Chaucer Review, 29, 4, p. 416-433.

DE GENDT Anne-Marie, 2003, L'art d'éduquer les nobles damoiselles : Le Livre du Chevalier de la Tour Landry, Paris, Champion.

Duby Georges, 1990, «Le modèle courtois », in Georges Duby \& Michelle PERrot (dir.), L’histoire des femmes en Occident: Le Moyen Age, Paris, Plon, p. 261-276.

KRUEGer Roberta L., 1993, "Intergeneric Combination and the Anxiety of Gender in Le Livre du Chevalier de la Tour Landry pour l'enseignement de ses filles ", L'Esprit Créateur, XXXIII, 4, p. 61-72.

—, 2005, «Identity Begins at Home: Female Conduct and the Failure of Counsel in Le Ménagier de Paris », Essays in Medieval Studies, XXII, p. 21-39.

MoI Toril, 2004, «From Femininity to Finitude: Freud, Lacan, and Feminism, Again », Signs: Journal of Women in Culture \& Society, 29, 3, printemps, p. 841-878.

RÉGNIER-BOHLER Danielle, 1993, "Galois, galoises et la nature du temps : Le monde bestourné chez le Chevalier de la Tour Landry ", in Jean-Claude Aubailly (dir.), Et c'est la fin pour quoy sommes ensemble. Hommage à Jean Dufournet. Littérature, histoire, et langue du Moyen Áge, Paris, Champion, p. 1187-1191.

Rose Christine M., 2002, "What Every Goodwoman Wants: The Parameters of Desire in Le Ménagier de Paris/The Goodman of Paris », Studia Anglica Posnaniensia, XXXVIII, p. 393-410.

UDRY Susan, 2002, «Robert de Blois and Geoffrey de la Tour Landry on Feminine Beauty: Two Late Medieval French Conduct Books for Women », Essays in Medieval Studies, XIX, p. 90-102. 